\title{
SENTINEL-2A BASED IMPERVIOUSNESS MONITORING OF URBAN BUILT ENVIRONMENT
}

\author{
Dyutisree Halder*, R. D. Garg \\ Dept. of Civil Engineering, Indian Institute of Technology, Roorkee, Uttarakhand, India- 247667
}

Commission III, WG III/II

KEY WORDS: Normalized Classification, Regression Modelling, SVM Classification, Impervious surface mapping

\begin{abstract}
:
The cities where the future happens first, they are open, creative, cosmopolitan and sexy and the perfect antidote to reactionary nationalism but the urbanization in unplanned manner is becoming an environmental-social-economical threat to accommodate the huge number of population which is literally boosting the present situation of climate change due to global warming. Extracting, measuring and treating the urban area which compiles of dense built-up and complex road network, is very essential to decrease the negative impact on environment. If most of the impervious surfaces can be replaced with permeable or semi-permeable materials or solar panel then the habitation will be saved from natural disastrous events like heat wave and flash flood. Urbanization can be categorized mainly into two: a) Static (urban open space + built space) and b) Dynamic (transportation). The static and dynamic urbanizations largely consist of impermeable or impervious materials. Impervious surfaces are alluded as the anthropogenic elements through that water can't infiltrate into the soil, such as streets, driveways, parking areas, houses, structures etc. An urban area is a densely populated human settlement, facilitated with multiple infrastructures including built and un-built. These areas or settlements are categorized as towns, suburbs, cities by urban morphology. Through balancing the ratio between the un-built (urban space) and built (building \& roads), urban disastrous events can be minimized. This research mainly focused on the extraction of impervious areas using regression modelling approach which is used to generate an impervious surface map from Sentinel-2A dataset of Delhi. Utilising multiple normalised indices can provide better classification results. This study shows that in urban areas imperviousness is becoming one of the prominent computational parameter and monitoring impervious areas could help us understand a lot of urban phenomena which are built-up induced and its rapid change in urban environment is giving rise to unhealthy living conditions.
\end{abstract}

\section{Introduction}

With a large number of individuals moving to urban areas, the urban region is growing at much faster rates to accommodate them. In past few years, handling this rapid urbanization has turned into a worldwide problem. In Asia, several large cities, for example, Dhaka, Delhi, Seoul and Beijing are home to more than 30 million individuals. Outside Asia, several large cities, for example, New York, Mexico City, Paris, London is facilitating more than 20 million individuals and the number is as yet boosting all along. Urbanization at all levels is an essential piece of advancement yet quick urbanization is raising the vulnerability of the region.

From the perspective of sociology, fast urbanization leads to several problems such as tight energy supply, traffic congestion, and increased crimes and so on. Urban percentage is only $3 \%$ of the earth surface but consumes $75 \%$ of world energy and produces $80 \%$ of GHGs (Green House Gas). On the other hand, some phenomenon such as urban flash flooding and heat island effects has become a threat to all the citizens living in the cities. Cities in the developing countries are more vulnerable to damages because their ability to adapt to catastrophes is restricted.

While urbanization is causing severe climate change to our earth which is affecting its environment at much faster ran than predicted. A total of 3 Million of population migrate to cities each week all over the world. But stopping migration isn't the solution, especially in countries like India where controlling population, balancing economics, equalization in society is a very tricky task. It will be best to create an urban environment that can synthesise with the natural environment and would cause less adverse effects or can assess the damage of existing urban environment to evaluate its restoration parameters.

*Corresponding Author

Email: dhalder@ce.iitr.ac.in
Urban environment mapping is an essential component for understanding the climate response in severe situation for future growth in sustainable way. This research aims at performing and evaluating impervious surface mapping on the basis of satellite images and evaluating the environmental problem caused due to increase in impervious percentage in Delhi. The solution it could provide in the form of betterment of urban surface understanding which could be helpful in the problem such as flash flood or pluvial flooding.

\section{Theoretical Background}

This research is based some of the basic understanding over the domain of urban built environment and how remote sensing could be useful for understanding these kind of spaces. This section gives an overview of the theoretical understanding of the concept used.

\subsection{Urban Built Environment and Imperviousness}

Settlements with dense infrastructure and dwellers of built environment, is defined as an urban space. These areas or settlements are created through intervention of human over natural land cover and are categorized as towns, conurbations or suburbs, cities by urban morphology and space which is an artefact of urbanization because it helps to improve the quality of life.

Impervious surfaces are alluded to as the anthropogenic elements through that water can't infiltrate into the soil, such as streets, driveways, parking areas, houses, structures etc. Impervious surfaces are significant to range of issues and themes in environmental science, so their knowledge especially the magnitude, location, geometry, spatial pattern and perviousness- 
imperviousness ratio is critical for this study and need to be justified.

Making impervious surfaces on the natural land prompts less water penetration into ground. Therefore, changing over pervious surfaces into impervious surfaces at a bigger scale may lead to a significant problems such as urban or pluvial flooding, urban heat island and flash flooding in a watershed or in the urban area located downstream, making a potential damage to the infrastructures and buildings in the urban areas. In addition, with less water penetrating into the ground in the long haul, the water cycling process in a watershed might be backed off and the water quality and water accessibility will lessened drastically.

Climate change is not a problem as it will occur anyway as the time passes by but the rate at which it is happening is the cause of distress and worry. Impervious Surface is responsible for these two effects of climate change; one is land surface temperature (LST) and second is increased runoff which helps to occur flood. So, identifying those surfaces in accurate percentage is important for management and treatment. This percentage varies with density, material, soil and other adjacent environment.

\subsection{V-I-S Model}

The theoretical foundation of impervious areas mapping with respective to medium resolution images is the V-I-S model proposed by Ridd (1995). Ridd assumed that the complex urban landscapes can simply be delineated as a mixture of vegetation, impervious surfaces and soil (V-I-S model) (Wang n.d.). In the urban territory, impervious areas, vegetation and soil have their own disseminated features. To be particular, impenetrable surface is spatially concentrated, high or low density residential and commercial areas. The vegetation segment basically contains grass and trees and they are for the most part dispersed in the suburban region or along channels or river. As for the soil, it may exist within and outside of the urban area and is a noteworthy wellspring of pervious surface. A number of studies on urban applications based on V-I-S model have been explored. It is described the urban composition of Cairo, Egypt as vegetation, impervious surface, soil, and shade, and consequentially applied the derived urban composition into detailed land use classification; Lu and Weng (2004) employed four components (green vegetation, impervious surface, soil and shade) to describe urban/rural environments. These studies have proven that the VI-S model based approach has potential to describe land covers and land uses in urban applications (Wang n.d.). Thus, the V-I-S model will be utilized as a part of this review to delineate impenetrable ranges in an urban area.

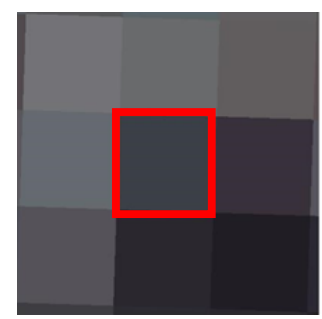

(a)

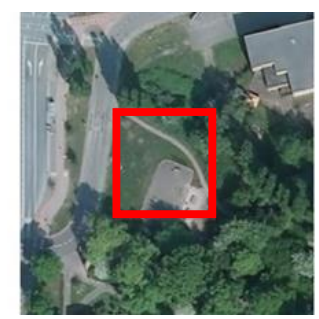

(b)
Figure 1. (a) Greenness of the rectangle: $0.027 \&$ NDVI of the rectangle: 0.052; (b) Visually Measured imperviousness percentage of the rectangle: $35 \%$ [Wang n.d.]

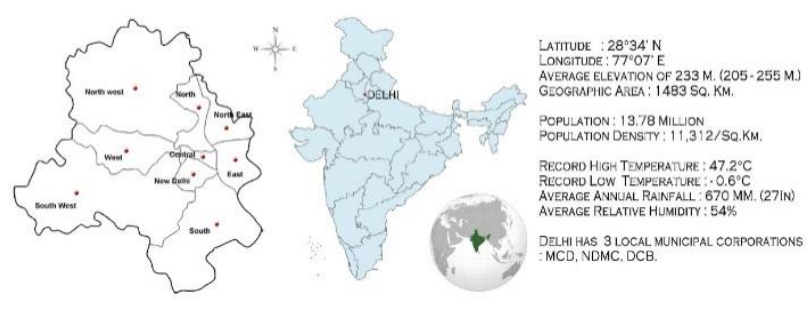

Figure 2. Location of Delhi

\section{Study Area}

Delhi is the national capital of India, the second most populated metro city with 17 million people in the world within the area of $1483 \mathrm{~km} 2$. In 1947, Delhi has population of 1.7 million and it has risen 1.7 to 16.8 (2011 census of India) in 2011, just in 60 years with density of $11,312 / \mathrm{km} 2$ where particularly the urban population is about 11 million (2011 Census). This is one of the fastest growing urban areas in history (United Nations, 2011), this rapid urbanisation with complexity of LULC with its huge heritage itself a very big challenge for researchers. Due to recent rapid and unplanned urbanization Delhi has been converted from a green patch to a concrete jungle. Due to this there has been a sudden or drastic changes in urban environment of Delhi.

\section{Methodology}

The methodologies utilized for impervious surface mapping with particular to medium and high spatial resolution are distinctive. The medium spatial resolution image is alluded to as an image with a pixel size of more than 30 meters, while the high spatial resolution image has a pixel of 10 meters. Simple pixel-based classification will prompt to inaccurate outcome to a huge degree due to impact of mixed pixel in coarse resolution. Rather, other than simple pixel-based classification, evaluating the extent of impervious surface inside one pixel is the best approach to delineate impervious areas for images with mixed pixel effect. Complete workflow which has been used in this study is shown in figure 3. Sentinel-2A data has been used for our study. In the process of delineating impervious areas, it's very important to understand the mass void ratio or the concept behind V-I-S model which is even very important in any urban/rural planning. While processing multispectral images we have large numerical variation in different band combination but in normalization, the range of pixel intensity value reduces and varies between -1 to 1 . 


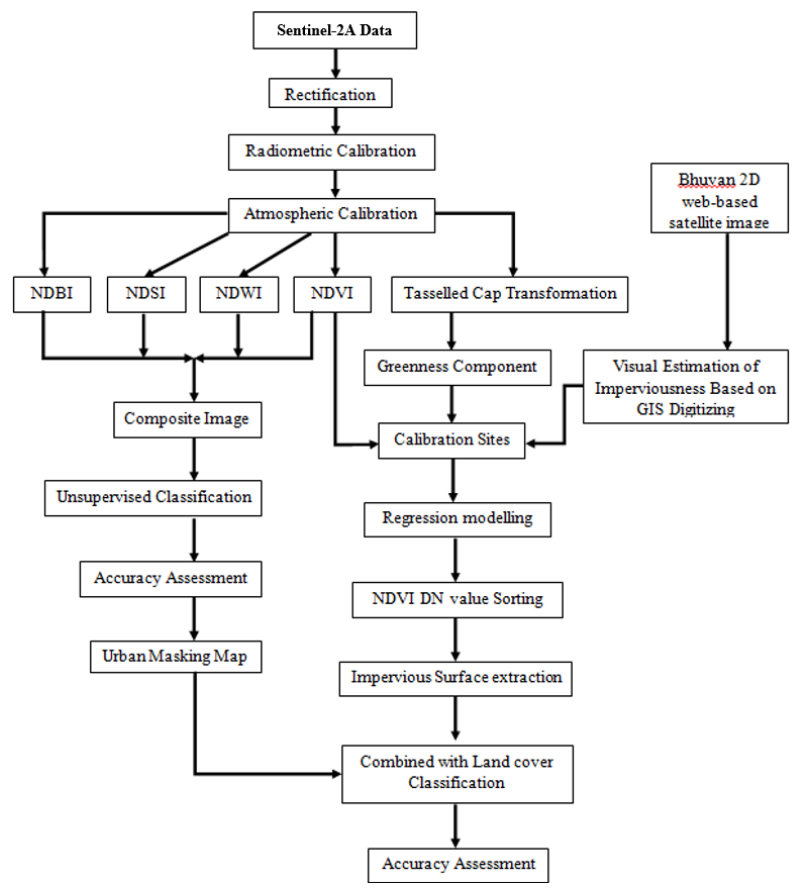

Figure 3. Methodology for Impervious Mapping

The NDVI (Normalized Difference Vegetation Index) is a standardized vegetation index which allows us to generate an image showing the relative biomass; the chlorophyll absorption in Red band and relatively high reflectance of vegetation in Near Infrared band. NDWI (Normalized Difference Water Index) has been developed to delineate open water features and enhance their presence in remotely sensed imagery based on reflected near-infrared radiation and visible green light. The NDSI (Normalized Difference Soil Index) is best to identify the fine line between built-up and bare soil in an urban area; correlation between MIR (Medium Infra-Red) and Green gives the highest. The NDBI (Normalized Difference Built-up Index) is considered to be given the best result showing the density in built massing (Deng et al. 2015). Multiple Normalised Images in stacked form provided some better classification result as compared to normal band definitions.

It is much more efficient to eliminate the non-urban areas and apply the regression modelling only to the urban areas. In the study area, pervious surfaces, such as forest and grassland, are mainly found in non-urban areas near the periphery of the city boundary whereas impervious surfaces, such as buildings, asphalts roads are mainly restricted within the urban areas. A mask of non-urban areas was created after executing classifications on the original images.

\section{Regression modelling approach}

The relationship between greenness and impervious surface percentage was extracted from 200 random ROI (Region of Interest) samples. For each sample, a pair of values of the greenness and actual impervious surface percentage was recorded. The actual imperviousness percentage value in each pixel of an image is estimated and calculated visually based on Bhuvan 2D satellite image and ground survey. The same procedure has been done with NDVI of same area and those same 200 samples were selected to identify the values.

A linear regression trend line relating percentage of impervious surface areas to the value of NDVI was determined from these 200 samples. Graph shown below illustrates the mathematical relationship between them. A second order regression trend line was determined. The correlation between percentage impervious surface and greenness is $80 \%$ (Fig 4a) indicating towards a strong relationship among them.

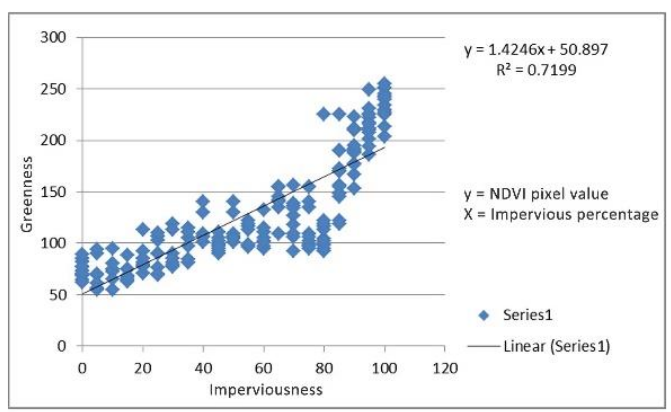

(a)

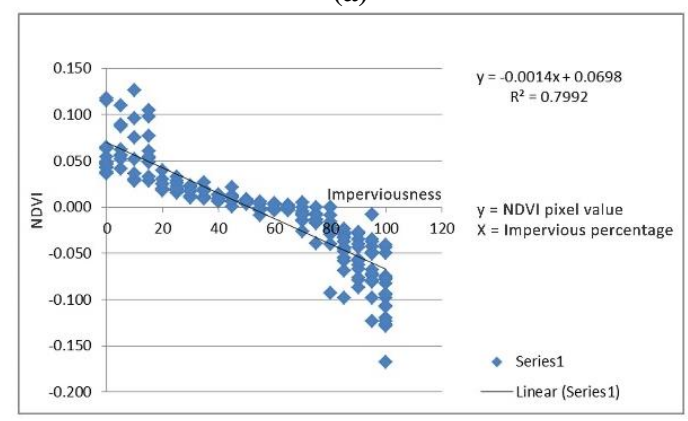

(b)

Figure 4. (a) Regression trend line for Greenness and imperviousness value; (b) Regression trend line for NDVI and imperviousness value

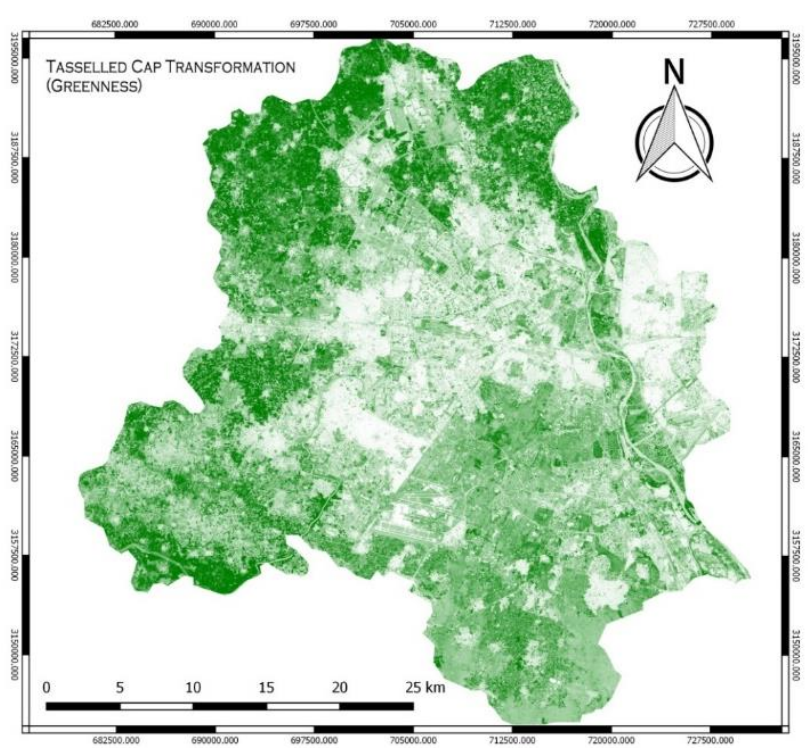

(a) 


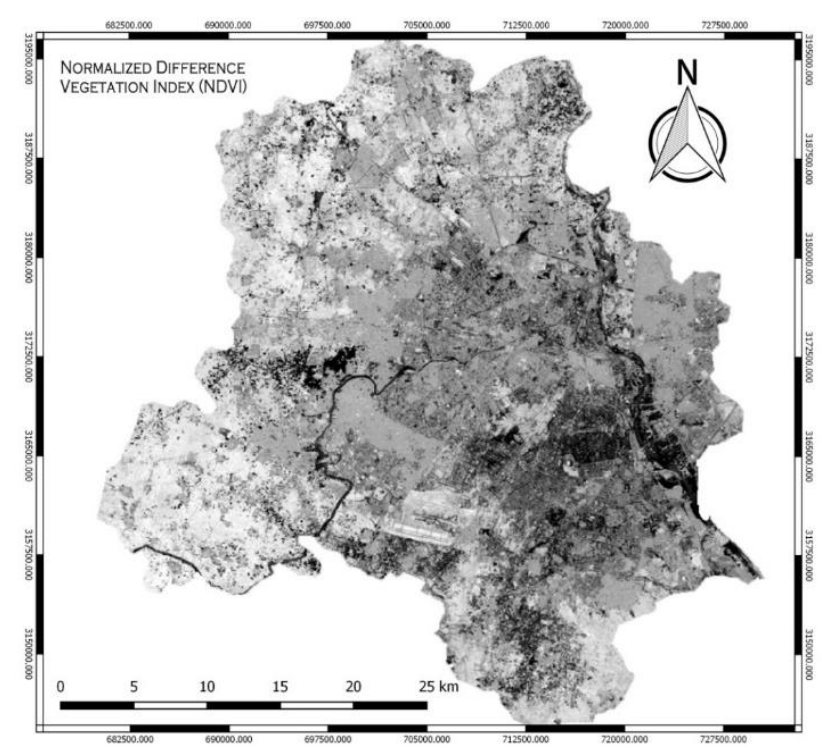

(b)

Figure 5. (a) Tasselled Cap transformation (Greenness Map);

6. Results

Imperviousness can be categorized as natural and artificial imperviousness depending upon its existence. Natural impenetrability considers regions with elevated surface runoff due to various soil types, surface properties and vegetation, while artificial impenetrability alludes to zones where changes in runoff amount are brought by human intervention. Extraction of exact amount of artificial imperviousness is very important for computing these surfaces runoff volumes and other effecting parameters. The normalized images were used to identify the specific properties individually and were stacked together which gave a combined glimpse of all the properties together. This reduced the time of supervised classification and gave satisfactory result as per accuracy assessment. Four land cover types were delineated and classified as vegetation, barren land, built-up and water based on stacked normalized image.

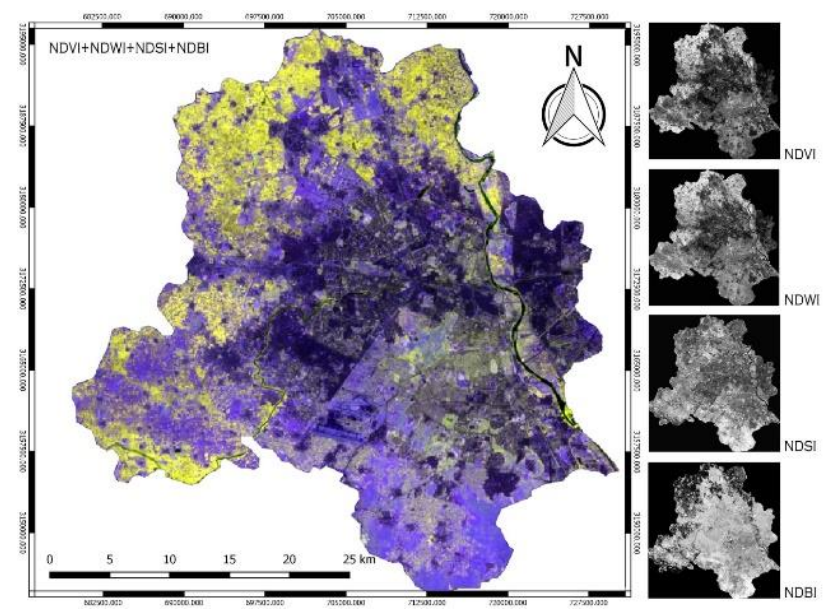

(a)

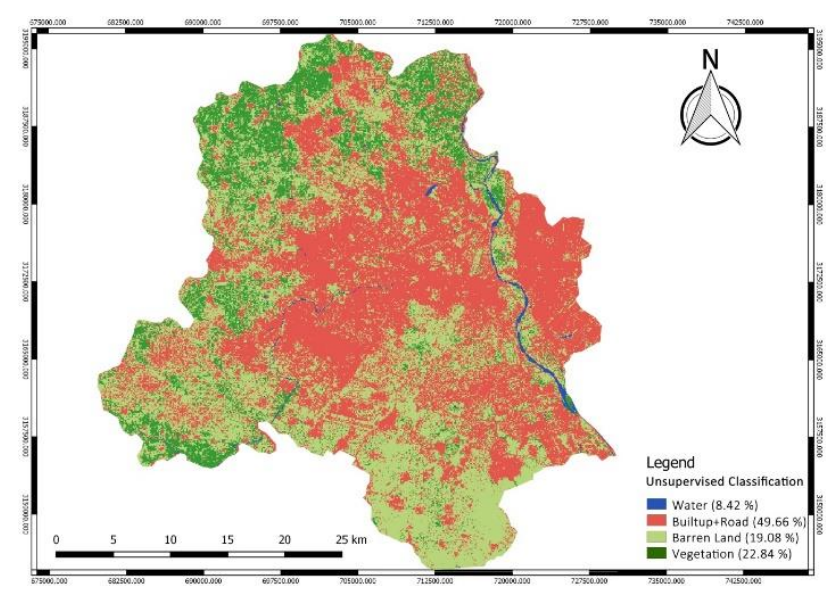

(b)

Figure 6. (a) Composite image stacked from NDVI, NDWI,

NDBI and NDSI; (b) Unsupervised classification using Normalized stacked Image

The non-urban area was masked so that the regression model was only applied to the urban area so that the distinction of impervious percentage can be well identified. This map gives the actual idea of mass and void ration in the city.

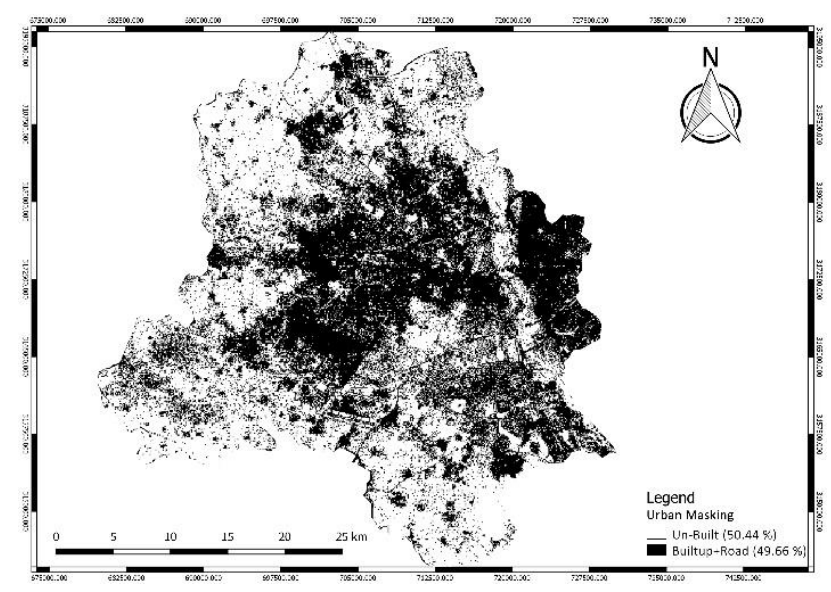

Figure 7. Urban Mask over study area

Previous research for impervious surface mapping has shown use of greenness component of tasselled cap transformation. So, following up with this fact that greenness can give us well understanding of imperviousness as it was assumed the inverse of greenness is imperviousness, it was found that the correlation between greenness pixel values and visual imperviousness was about $72 \%$. Whereas uni-dimension spectral indices such as NDVI and EVI perform better at extremely high or low level of covers. 


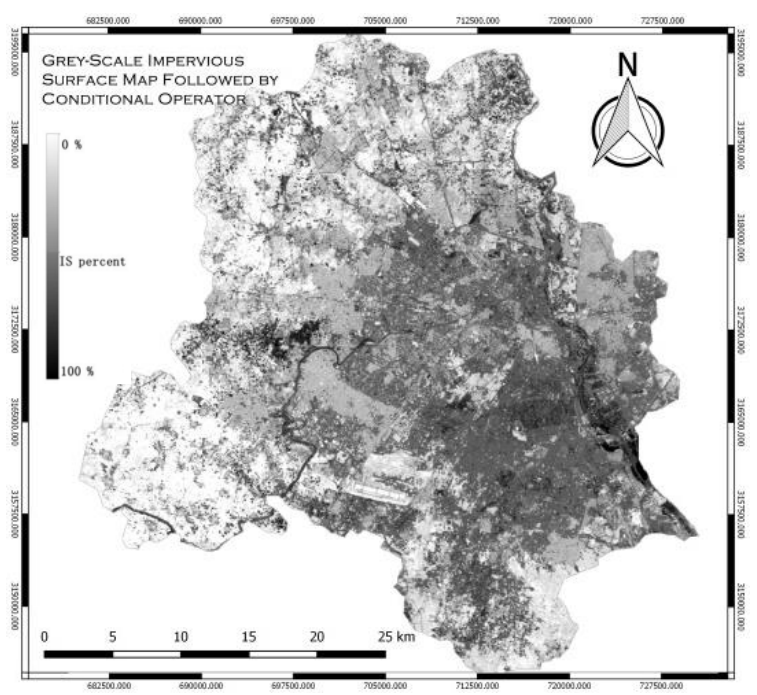

Figure 8. Impervious surface mapping through Regression modelling

\begin{tabular}{|c|c|r|c|c|}
\hline NDVI Pixel Values & Colour & $\begin{array}{c}\text { Pixel Counts } \\
(\mathbf{3 0 m} \mathbf{~ X ~ 3 0 m )})\end{array}$ & $\begin{array}{c}\text { Ares } \\
\left(\mathbf{k m} .{ }^{2}\right)\end{array}$ & Imperviousness \% \\
\hline$>(0.202)$ & $255,255,255$ & 38 & 0.03 & $<10$ \\
\hline$(0.202)-(0.055)$ & $227,227,227$ & 220,066 & 198.06 & $10-20$ \\
\hline$(0.055)-(0.027)$ & $199,199,199$ & 287,244 & 258.52 & $20-30$ \\
\hline$(0.027)-(0.007)$ & $171,171,171$ & 268,509 & 241.66 & $30-40$ \\
\hline$(0.007)-(-0.009)$ & $143,143,143$ & 237,577 & 213.82 & $40-50$ \\
\hline$(-0.009)-(-0.015)$ & $112,112,112$ & 64,181 & 57.76 & $50-60$ \\
\hline$(-0.015)-(-0.099)$ & $84,84,84$ & 484,536 & 436.09 & $60-70$ \\
\hline$(-0.099)-(-0.141)$ & $56,56,56$ & 82,785 & 74.51 & $70-80$ \\
\hline$(-0.141)-(-0.220)$ & $28,28,28$ & 32,009 & 28.81 & $80-90$ \\
\hline$<(-0.220)$ & $0,0,0$ & 4,083 & 3.67 & $>90$ \\
\hline
\end{tabular}

Figure 9. Impervious area with corresponding Imperviousness percentage

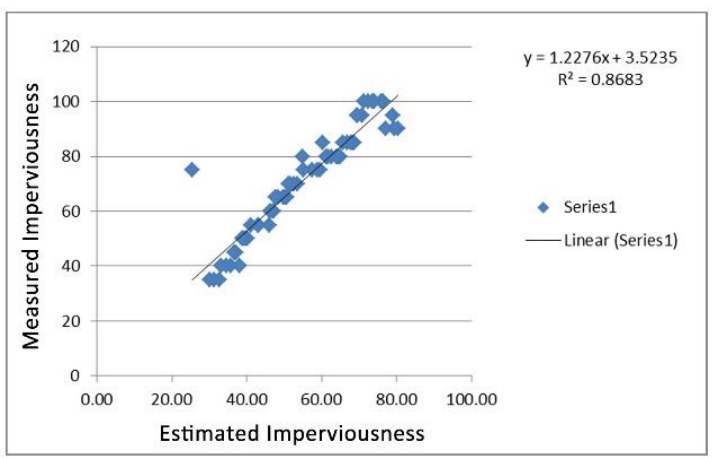

Figure 10. Relation between Measured and Estimated Impervious Surface Percentage

Another interesting observation which showed us that natural impervious surface also take part in impervious surface mapping which is very important. During monsoon season when already the water bodies, wet lands have reached their $80 \%-100 \%$ saturation point and even barren lands start act as impervious surfaces as per their soil property and infiltration rates.

From the accuracy assessment using 60 samples it can be concluded that the agreement is high with a $\mathrm{R}^{2}$ of 0.87 which is applied only on urban area. In addition, the RMSE value was calculated as $17.82 \%$, indicating a promising agreement between estimated and measured imperviousness. In Urban areas imperviousness is becoming one of the prominent computational parameter, as rapid change in urban environment is giving rise to unhealthy living conditions.

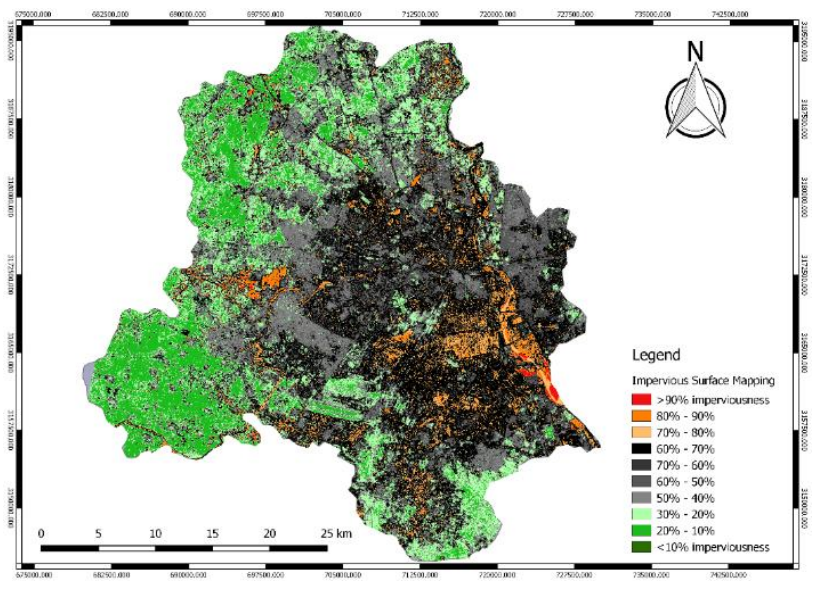

Figure 11. Impervious Surface Map of Delhi

\section{Discussion}

This research mainly focused on the extraction of impervious areas using regression modelling approach which is used to generate an impervious surface map. Which helped us to assess the risk caused due to this increment in urban environment using Sentinel-2A data of Delhi region and unsupervised classification were used to delineate mostly urban and non-urban features.

Mapping of urban environment is very much critical to understand the present scenario of an urban area and this will also help in predicting the future conditions. Imperviousness is one of several important parameters that are responsible for rapid change in urban environments. These changes are making cities and urban areas uninhabitable. As a future scope of this paper role of all other parameters will be evaluated through further investigation.

Imperviousness is very crucial part of surface mapping in urban area because not only artificial surfaces even natural impervious surfaces also play a very important role in it. Natural impervious surfaces change their characteristics with the time; barren land, wet land, sandy beach and water body act based on their moisture contains in monsoon and solar reflectance in summer or winter season. During this research, it was experienced that images of summer season give better results of analysing the artificial impervious surfaces whereas images of monsoon season give better analysis of natural impervious surfaces. As Delhi is densely populated area with massive built-up; so the image was considered to be the best dated on 7th May 2016 after several temporal image processing. But rather than that the images of summer, both monsoon and summer data could be merged after processing and rectification would be the best.

For further detailed mapping, images with higher spatial and spectral resolution can be used with respect to identify the confliction between artificial and natural impervious surfaces using further classification. It is recommended to investigate a more efficient method to minimize the observed underestimation in the built-up areas using Normalized Linear Spectral Mixing Analysis (NLSMA) method. As high spatial resolution images are becoming more available, classifications based on objectbased image analysis (OBIA) are promising, it utilizes the spectral and contextual information to extract land use and land cover. 


\section{Conclusions}

Various approaches have been shown for justifying the urban environment through impervious surface extraction. The unsupervised classification was processed to give almost accurate classes which were done on the stacked image of the four normalized indices. Overall accuracy was evaluated more than $85 \%$. The mapping is done using images with $10 \mathrm{~m}$ resolution. Images were enriched with large number of mixed pixels but unidirectional NDVI was found to have better correlation than greenness out of other three dimensional components.

Regression modelling approach also achieved an accuracy of $87 \%$ which indicates it has a high agreement with the real landscapes. The extracting percentage of the imperviousness of the city was quite accurate which can be determined through the accuracy assessment and this will be helpful for further stage of resilient urban planning against all effects of climate change.

\section{REFERENCES}

Analysis, G. (n.d.). Impervious Surface Mapping Using Satellite Remote Sensing, 1-4.

Bauer, M. E., Doyle, J. K., \& Heinert, N. J. (2002). Impervious Surface Mapping using Satellite Remote Sensing, 0(C), 23342336.

Bhawan-ii, V., \& Road, B. (2014). Statistical Abstract Statistical abstract of Delhi.

Boffet, A., \& Coquerel, C. (2000). Urban classification for generalization orchestration. International Archives of Photogrammetry and Remote Sensing, 33(B4/1; PART 4), 132 139.

Breitspiel, I. \& Fon, H., Hazard of Pluvial Flooding A danger foreseen is a danger avoided !, 49(0).

Civco, D., Chabaeva, A., \& Hurd, J. (n.d.). A Comparison of Approaches to Impervious Surface Characterization, 1-5.

Cruz, S. (n.d.). Impervious Surface Coefficients, 10-13.

Dash, P., Verma, N., \& Aggarwal, S. P. (2013). Effect Of Land Use Change On Surface Runoff In An Ungauged Basin In Western Himalaya, India.

Deng, Y. et al., 2015. International Journal of Applied Earth Observation and Geoinformation RNDSI : A ratio normalized difference soil index for remote sensing of urban / suburban environments. International Journal of Applied Earth Observations and Geoinformation, 39, pp.40-48. Available at: http://dx.doi.org/10.1016/j.jag.2015.02.010.

Developer, D., Architect, D., \& Server, E. (2009). Impervious Surface Mapping with Definiens, 2009, 0-3.

Faster, M. W. (n.d.). How Urbanization Affects the Water Cycle, 3-6.

Government, H. A. (n.d.). Disaster Management Ministry of Home Affairs.
Impervious, N. D., \& Xu, H. (2017). Analysis of Impervious Surface and its Impact on Urban Heat Environment using the Analysis of Impervious Surface and its Impact on Urban Heat Environment using the Normalized Difference Impervious Surface Index (April).https://doi.org/10.14358/PERS.76.5.557

(NDISI),

Journal, I., \& Geomatics, O. F. (2012). Population calibrated land cover impervious surface coefficients for Upper Bhima basin, 2(4), 1027-1047.

Journal, I., \& Sensing, R. (2016). Use of normalized difference built-up index in automatically mapping urban areas from TM imagery,

(February

https://doi.org/10.1080/01431160304987

Li, S., \& Chen, X. (2014). A New Bare-Soil Index For Rapid Mapping Developing Areas Using Landsat 8 Data, Xl(May), 1416. https://doi.org/10.5194/isprsarchives-XL-4-139-2014

Lin, H. (2013). A comparison study of impervious surfaces estimation using optical and SAR remote sensing images. International Journal of Applied Earth Observations and Geoinformation, 18(December), 148-156. https://doi.org/10.1016/j.jag.2011.12.015

Mallick, J., \& Rahman, A. (2012). Impact of population density on the surface temperature and micro-climate of Delhi, 102(12).

Practices, B. M., \& States, U. (2015). Impervious Surface Mapping, 0-1.

Schug, F., Furian, W., \& Schug, F. (n.d.). Mapping impervious urban surfaces using regression analysis on synthetic hyperspectral EnMAP data Mapping impervious urban surfaces using regression analysis on synthetic hyperspectral EnMAP data.

Series, S. L. (2009). Permeable vs . Impermeable Surfaces, (January).

Sexton, J. O., Song, X., Huang, C., Channan, S., Baker, M. E., \& Townshend, J. R. (2013). Remote Sensing of Environment Urban growth of the Washington, D . C . - Baltimore, MD metropolitan region from 1984 to 2010 by annual, Landsat-based estimates of impervious cover. Remote Sensing of Environment, 129, 42-53. https://doi.org/10.1016/j.rse.2012.10.025

Surface, I., \& Tool, A. (n.d.). ISAT : Impervious Surface Analysis Tool.

Sustainable, F., \& Management, S. (n.d.). The following list of Low Impact Development (LID) and Green Infrastructure techniques are examples of strategies that can be employed to control stormwater runoff in Hudson County. In practice, these techniques utilize engineered or natural systems to mimic natural processes and pre - the suitability of a strategy or project will depend on the physical benefits of the strategy. Physical factors vary with respect to runoff rate control, redevelopment projects, the use of should be considered a priority tool for, (Lid), 1-10.

Taati, A., Sarmadian, F., \& Mousavi, A. (2014). Land Use Classification using Support Vector Machine and Maximum 
Likelihood Algorithms by Landsat 5 TM Images. Walailak Journal of, 12(8), 681-687. Retrieved from http://wjst.wu.ac.th/index.php/wjst/article/view/1225

Taubenböck, H., Esch, T., \& Roth, A. (2006). an Urban Classification Approach Based on an Object - Oriented Analysis of High Resolution Satellite Imagery for a Spatial Structuring Within Urban Areas, (March), 2-3.

The, U. F. O. R., Ecotoxicology, O., Integrated, P., Assessment, R., Office, B., Health, E., \& Assessment, H. (n.d.). U ' g c i s c.

US National Research Council. (2011). Warmıng World Impacts by Degree, 40 .

Uvm, L. A. M., Uvm, K. P., Callahan, J., Anr, V. T., Csb, H. S., \& Csb, T. D. (2006). Mapping Impervious Surfaces with High Resolution QuickBird Satellite Data, 17.

Wang, Y., Impervious surface mapping using satellite data and runoff modelling in Amersfoort, NL.

Water, N., Inventory, Q., \& Epa, U. S. (1996). 4.0 Environmental Assessment, 1-49.

Weng, Q. (2001). Modeling Urban Growth Effects on Surface Runoff with the Integration of Remote Sensing and GIS, 28(6), 737-748. https://doi.org/10.1007/s002670010258

Zhang, H., Chen, Y., \& Zhou, J. (2015). Assessing the long-term impact of urbanization on run-off using a remote-sensingsupported hydrological model. International Journal of Remote Sensing, $36(21)$, $5336-5352$. https://doi.org/10.1080/01431161.2015.1094834 